\title{
Prompt spectral properties of a complete sample of Swift Gamma-Ray Bursts
}

\section{Lara Nava*1, Ruben Salvaterra ${ }^{2}$, Giancarlo Ghirlanda ${ }^{3}$, Gabriele Ghisellini ${ }^{3}$, Sergio Campana $^{3}$, Stefano Covino ${ }^{3}$, Paolo D'Avanzo ${ }^{3}$, Andrea Melandri ${ }^{3}$, Boris Sbarufatti ${ }^{3}$, Gianpiero Tagliaferri ${ }^{3}$ and Susanna D. Vergani ${ }^{3}$}

${ }^{1}$ APC, Univ Paris Diderot, CNRS/IN2P3, CEA/Irfu, Obs de Paris, Sorbonne Paris Cité, 10, rue Domon et Duquet, 75205 Paris, France

2 INAF, IASF Milano, via E. Bassini 15, I-20133 Milano, Italy

${ }^{3}$ INAF, Osservatorio Astronomico di Roma, Via Frascati 33, 00040, Monte Porzio Catone, Rome, Italy

E-mail: lara.nava@apc.univ-paris7.fr

\begin{abstract}
Thanks to Swift, rapid follow-up studies of GRBs are possible. About 1/3 of all GRBs observed by Swift has measured redshift (an enormous improvement with respect to the pre-Swift era). Salvaterra et al recently defined a subsample of Swift GRBs with $90 \%$ of redshift completeness, based on favorable observing conditions (Jakobsonn et al) and prompt brightness. We present their spectral properties and for the first time investigate the spectral-energy correlations in a sample unaffected by biases induced by the measurement of $z$. Using the complete sample as reference, we simulate samples of GRBs to probe the role of the flux threshold on the $E_{\text {peak }}-L$ relation. We found that the use of flux limited samples in correlation studies cannot be responsible for the existence of the correlation itself.
\end{abstract}

Gamma-Ray Bursts 2012 Conference-GRB2012,

May 07-11, 2012

Munich, Germany

\footnotetext{
* Speaker.
} 


\section{Introduction}

The measure of the distance of Swift bursts has been secured for only $\sim 1 / 3$ of the cases. The low completeness level in redshift determination, strongly limits the possibility of well grounded statistical studies of the rest-frame properties of long GRBs. Therefore, it is important to obtain an unbiased complete sample of GRBs, capable to fully represent this class of object. To this end, we select bursts that have favorable observing conditions for redshift determination from ground and that are bright in the $15-150 \mathrm{keV}$ Swift/BAT band. We find 58 bursts matching our selection criteria with a completeness level in redshift determination of $90 \%$ ( $~ 95 \%$ if we consider also the redshift constraints imposed by the detection of the afterglow or host galaxy in some optical filters). Our sample provides the solid basis for the study of the long GRB population in an unbiased way. We take advantage of the high completeness level of our sample to constrain the GRB luminosity function (Salvaterra et al. 2012), to study the correlation between physical parameters of the bursts (Nava et al. 2012; D'Avanzo et al. 2012), the properties of the burst light curves and of the environment in which they explode (Campana et al. 2012; Melandri et al., 2012).

In this paper we present the main results about the luminosity function (and its possible evolution with cosmic time) and then we focus on the study of spectral-energy correlations. In particular, we investigate the correlation between the rest frame peak energy $E_{\text {peak }}$ of the $v F_{v}$ prompt spectrum and the isotropic energy $E_{\text {iso }}$ (Amati et al. 2002), and the correlation between $E_{\text {peak }}$ and the isotropic peak luminosity $L_{\text {iso }}$ (Yonetoku et al. 2004). These correlations are valid for long GRBs. There are indications that short bursts obey the very same $E_{\text {peak }}-L_{\text {iso }}$ relation defined by long events but they are inconsistent with the $E_{\text {peak }}-E_{\text {iso }}$ correlation (Ghirlanda et al. 2009).

Despite the considerably large number of bursts consistent with these correlations, their physical origin is debated. Some authors claim that they are the result of instrumental selection effects (Nakar \& Piran 2005; Band \& Preece 2005). Other studies (Ghirlanda et al. 2008; Nava et al. 2008) quantified the possible instrumental selection biases finding that, even if they do affect the sample, they cannot be responsible for the existence of the spectral-energy correlations. GRBs added to the $E_{\text {peak }}-E_{\text {iso }}$ and $E_{\text {peak }}-L_{\text {iso }}$ correlations need to have their redshifts measured. This raised the suspect that these correlations might be biased by the high level of incompleteness in redshift of the samples of GRBs so far used. In this paper we investigate this issue with our sample, which is nearly complete in redshift.

Finally, we take advantage of our complete sample to study the impact of instrumental selection effects on the $E_{\text {peak }}-L_{\text {iso }}$ correlation. In particular, we answer to this specific question: might the $E_{\text {peak }}-L_{\text {iso }}$ correlation be produced by the threshold of a flux-limited sample of bursts?

\section{The sample}

Jakobsson et al. (2006) proposed a series of criteria in order to carefully select long GRBs which have observing conditions favorable for redshift determination. While none of their criteria is expected to alter significantly the redshift distribution of observed GRBs, the completeness level is increased to $\sim 53 \%$.

To obtain a more complete sample we select bursts matching the above criteria and having 1-s peak photon flux $P \geq 2.6 \mathrm{ph} \mathrm{s}^{-1} \mathrm{~cm}^{-2}$. Up to May 2011, 58 GRBs match our selection criteria 


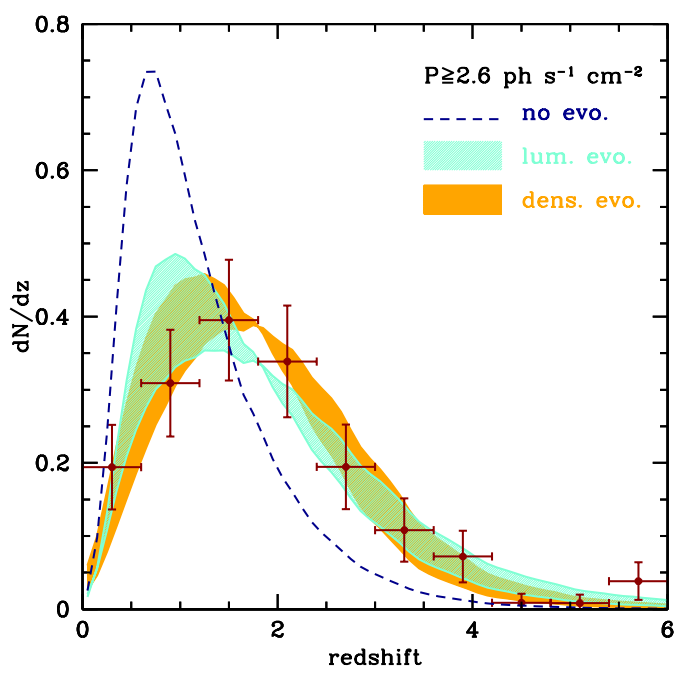

Figure 1: Normalized redshift distribution of the complete sample (red data points). Error bars show the Poisson uncertainties on the number of detection in the redshift bin. The dashed line shows the expected distribution for the no-evolution case. Results of luminosity and density evolution models are shown with the light blue and dark shaded orange regions, respectively, taking into account the errors on the evolution parameter.

and are listed in Table 1 of Salvaterra et al. 2012 (S12). 52 of them have measured redshift so that our completeness level is $90 \%$. Moreover, for 3 of the 6 bursts lacking measured $\mathrm{z}$ the afterglow or the host galaxy have been detected in at least one optical filter, so that $\sim 95 \%$ of the bursts in our sample have a constrained redshift.

For 46 bursts (out of 58) we can estimate $E_{\text {peak }}, L_{\text {iso }}$ and $E_{\text {iso. }}$. For 6 GRBs, instead, there is a good knowledge of the prompt spectral shape (i.e. $E_{\text {peak }}^{\text {obs }}$ is well constrained), but the redshift is unknown (in 3 cases it is possible to derive an upper limit on $z$ from the photometry of the afterglow or of the host galaxy). For the remaining 6 bursts the determination of $E_{\text {peak }}^{\text {obs }}$ is not possible: in 3 cases the spectrum is well described by a single power-law, while in the other 3 cases it is possible to set a lower limit to the observed value of $E_{\text {peak }}^{\text {obs }}$. Spectral properties of the 58 GRBs of the complete sample are reported in Table 1 of Nava et al. 2012 (N12).

It is interesting to compare the $E_{\text {peak }}-E_{\text {iso }}$ and $E_{\text {peak }}-L_{\text {iso }}$ correlations defined by the complete sample with the same correlations defined with a larger sample of GRBs, comprising all bursts with measured redshifts and $E_{\text {peak }}^{\text {obs }}$ detected by different satellites. This sample (called in the following the 'total' sample) up May 2011 contains 136 bursts.

\section{Redshift distribution and luminosity function}

The redshift distribution of the bursts in our sample is shown in Fig. 1. The mean (median) redshift of the sample is $1.84 \pm 0.16(1.64 \pm 0.10)$ with a long tail at high- $z$ extending, at least, up to $z=5.47$. The expected redshift distribution of GRBs can be computed once the GRB LF and the GRB formation history has been specified. We explore two general expression for the GRB LF: a single power-law with an exponential cut-off at low luminosity and a broken power- 


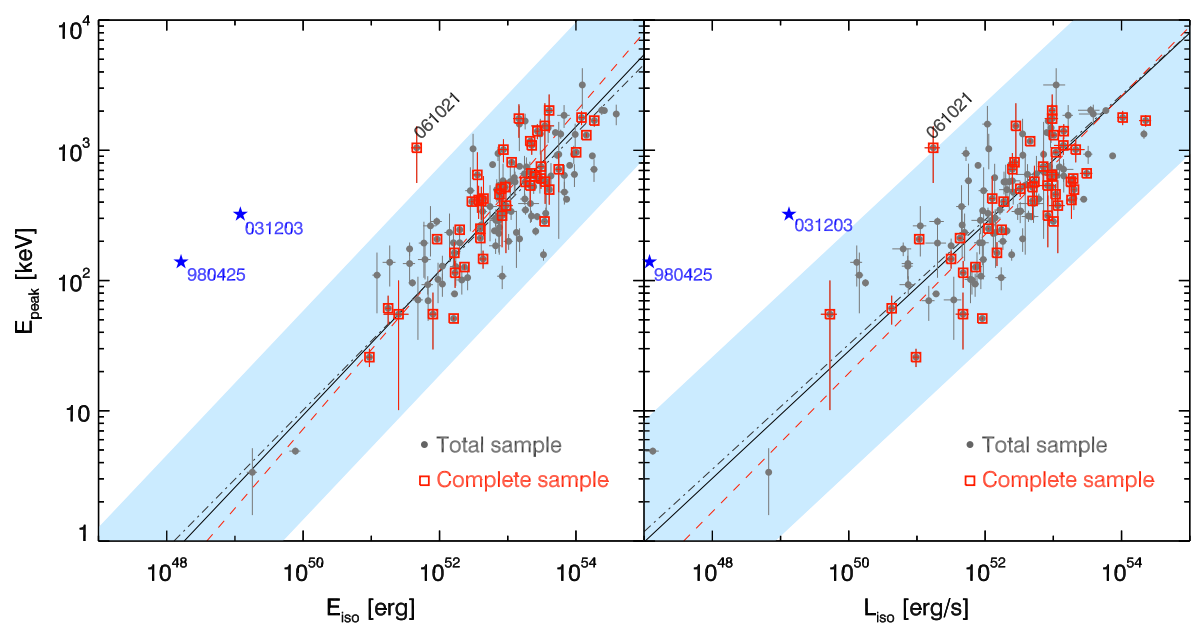

Figure 2: Amati (left panel) and Yonetoku (right panel) correlations. Grey filled circles refer to the total sample. Their power-law fit is shown as a solid dark line. Bursts belonging to the complete sample are marked as empty red squares and the red dashed line represents their best fit model. The position of the two historical outliers, GRB 980425 and GRB 031203, is also shown (blue stars), together with the burst of our complete sample, GRB 061021, that is above the $3 \sigma$ scatter limit.

law LF. We optimize the value of the model free parameters by minimizing the C-stat function jointly fitting the observed differential number counts in the 50-300 keV band of BATSE and the observed redshift distribution of bursts in our sample. The corresponding redshift distributions are shown in Fig. 1. The simple no-evolution scenario (dashed line in Fig. 1) does not provide a good representation of the observed redshift distribution of our sample: the rate of GRBs at high- $z$ is underpredicted. For this reason, we consider evolution scenarios that may enhance the number of detections at high- $z$. If the cut-off (break) luminosity is an increasing function of the redshift (that is $L_{\text {cut }}(z)=L_{\text {cut }, 0}(1+z)^{\delta_{l}}$ ) then high- $z$ GRB are typically brighter than low- $z$ bursts (luminosity evolution model). We find that a strong luminosity evolution with $\delta_{l}=2.3 \pm 0.6$ is required to reproduce the observed redshift distribution of the bursts in our complete sample (light shaded area in Fig. 1). Also density evolution models may lead to an enhancement of the GRB formation rate with redshift. We parametrize the evolution in the GRB formation rate as $\eta(z)=\eta_{0}(1+z)^{\delta_{n}}$. By fitting our datasets we find that strong density evolution is required with $\delta_{n}=1.7 \pm 0.5$.

\section{4. $E_{\text {peak }}-E_{\text {iso }}$ and $E_{\text {peak }}-L$ correlations and evolution with redshift}

We test the $E_{\text {peak }}-E_{\text {iso }}$ and the $E_{\text {peak }}-L_{\text {iso }}$ correlations with the sample of 46 bursts (included in the complete sample) with firm estimates of the redshift and of the spectral properties. The results are shown in Fig. 2. Data points from the total (incomplete) sample are plotted for comparison as grey dots. Red squares show the bursts of the complete sample. We found $E_{\text {peak }} \propto E_{\text {iso }}{ }^{0.61}$ (rank's correlation coefficient $\rho=0.76$ and chance probability $\left.\mathrm{P}_{\text {chance }}=7 \times 10^{-10}\right)$ and $E_{\text {peak }} \propto L_{\text {iso }} 0.53$ $\left(\rho=0.65\right.$ and $\left.\mathrm{P}_{\text {chance }}=1 \times 10^{-6}\right)$.

The behavior of GRB 061021 is very peculiar, since it lies at more than the $3 \sigma$ from the Amati and Yonetoku correlations. Twelve events belonging to the complete sample cannot be directly 

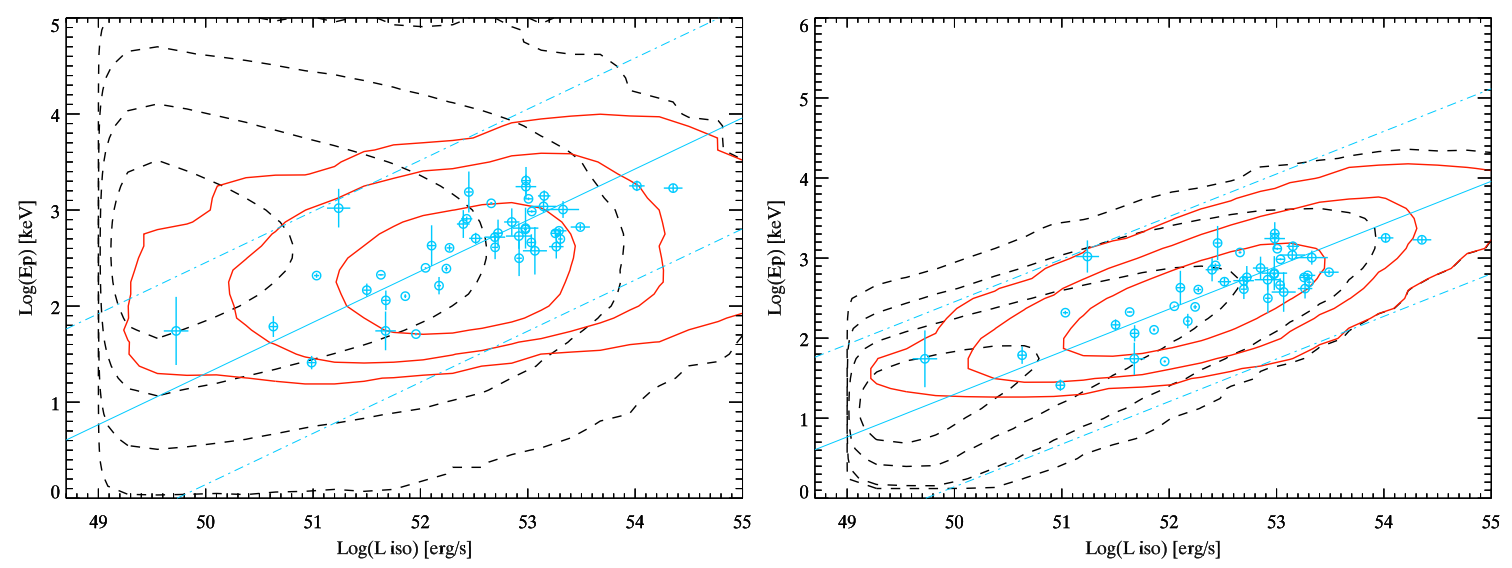

Figure 3: The simulated population of GRBs is shown by the dashed contours (1, 2, 3 and $4 \sigma$ levels). Those with a flux in the 15-150 keV energy range larger than the flux limit $F_{\text {lim }}=2.6 \mathrm{ph} \mathrm{cm}^{-2} \mathrm{~s}^{-1}$ of the Swift complete sample are shown by the (red) solid contours. The open (blue) circles are the 46 GRBs of the complete Swift sample. Left panel: no intrinsic correlation between $E_{\text {peak }}$ and $L_{\text {iso }}$ is assumed. Right panel: the simulation is performed by assuming a relation between $E_{\text {peak }}$ and $L_{\text {iso }}$.

used to test the correlations, since they have some unknown property: the redshift or the spectral peak energy. However, we tested their consistency with the correlations and we found that they are all consistent.

The possibility that evolutionary effects play a role in the spectral-energy correlations still represents an open issue. To investigate if the slopes of the $E_{\text {peak }}-E_{\text {iso }}$ and $E_{\text {peak }}-L_{\text {iso }}$ correlations have a dependence on the redshift we define 4 bins of redshift and investigate the correlations in each bin. There is no evidence of a systematic evolution of the slope with $z$.

\section{The impact of selection biases on the $E_{\text {peak }}-L$ correlation}

We performed Monte Carlo simulations of GRBs with a given redshift distribution and luminosity function (as described in Ghirlanda et al., 2012). From the simulated sample we extract a subsample of GRBs that have a peak flux $\geq F_{\text {lim }}=2.6 \mathrm{ph} \mathrm{cm}^{-2} \mathrm{~s}^{-1}$ (which is the same flux limit of the complete Swift sample studied in S12 and N12). We also required that $E_{\text {peak }}^{\text {obs }}$ of the detected bursts can be measured by current instruments, i.e. that it lies in the $15 \mathrm{keV}-2 \mathrm{MeV}$ energy range. These are the simulated bursts that would be detected by Swift.

If we make the hypothesis that there is no correlation between $E_{\text {peak }}$ and $L_{\text {iso }}$, only in $7.3 \%$ of the repeated simulations (e.g. for the case of a GRB population evolving in density with redshift, as found in S12) we find a statistically robust (i.e. chance probability of the rank correlation coefficient $\leq 10^{-3}$ ) $E_{\text {peak }}-L_{\text {iso }}$ correlation (figure 3). If we also require that our simulations produce a correlation similar (in slope, normalization and scatter) to that observed among real GRBs of the complete Swift sample, the percentage reduces to $0.7 \%$. Therefore, we reject the null hypothesis that there is not an intrinsic $E_{\text {peak }}-L_{\text {iso }}$ correlation at the $2.7 \sigma$ level of confidence $(3.0 \sigma$ for the case of luminosity evolution). 


\section{Conclusions}

We select a sub-sample of Swift long GRBs that is complete in redshift. The sample is composed by bursts with favorable observing conditions and with 1-s peak photon fluxes $P \geq 2.6 \mathrm{ph}$ $\mathrm{s}^{-1} \mathrm{~cm}^{-2}$. It contains 58 bursts with a completeness level of $\sim 90 \%$.

We use the observed burst redshift distribution of our complete sample to probe and constrain the evolution of the long GRB population in redshift. We confirm that GRBs must have experienced some sort of evolution being more luminous or more numerous in the past than observed today.

We use this complete sample to study the spectral-energy correlations in an unbiased way. We find that this sample defines two strong correlations: (i) $E_{\text {peak }}-E_{\text {iso }}$ (rank's correlation coefficient $\rho=0.76$ and chance probability $\left.\mathrm{P}_{\text {chance }}=7 \times 10^{-10}\right)$ and (ii) $E_{\text {peak }}-L_{\text {iso }}(\rho=0.65$ and $\left.\mathrm{P}_{\text {chance }}=1 \times 10^{-6}\right)$. The slope are 0.61 and 0.53 for the Amati and the Yonetoku correlations, respectively. These slopes are consistent within $1 \sigma(2 \sigma)$ with the slopes of the $E_{\text {peak }}-L_{\text {iso }}$ $\left(E_{\text {peak }}-E_{\text {iso }}\right)$ correlation defined by bursts not satisfying the completeness criteria (complementary sample). Our study outlines the presence of one GRB (061021) that lies at $3 \sigma$ limit (or more) of the tested correlations. In particular, it is an outlier to the Amati correlation. The presence of outliers to the Amati relation is somehow expected from the study of the observational plane $E_{\text {peak }}^{\text {obs }}-$ Fluence. Nava et al. $(2008 ; 2011)$ studied the distribution of BATSE and GBM bursts in this plane and derived respectively a $6 \%$ and $3 \%$ of outliers. By taking advantage of our complete sample, for both correlations we investigate the possible evolution with the redshift of the best fit slopes. To this aim we define 4 bins of redshift, chosen in order to have 4 sub-samples characterized by a similar number of objects. We find no relation between the slope of each sub-sample and the redshift.

We investigate the role of instrumental biases on the $E_{\text {peak }}-L_{\text {iso }}$ correlation. Our simulations show that the this correlation cannot be due to a selection bias caused by a flux-limited sample. These results suggest that a correlation between $L_{\text {iso }}$ and $E_{\text {peak }}$ should have a physical origin.

\section{References}

[1] D.L. Band \& R.D. Preece, Testing the Gamma-Ray Burst Energy Relationships, ApJ 627 (2005) 319.

[2] S. Campana, R. Salvaterra, A. Melandri, et al., The X-ray absorbing column density of a complete sample of bright Swift gamma-ray bursts, MNRAS 421 (2012) 1697.

[3] P. D’Avanzo, et al., MNRAS submitted.

[4] G. Ghirlanda, L. Nava, G. Ghisellini, C. Firmani, J.I. Cabrera, The $E_{\text {peak }}-E_{\text {iso }}$ plane of long gamma-ray bursts and selection effects, MNRAS 387 (2008) 319.

[5] G. Ghirlanda, L. Nava, G. Ghisellini, A. Celotti, C. Firmani, Short versus long gamma-ray bursts: spectra, energetics, and luminosities, A\&A 496 (2009) 585.

[6] G. Ghirlanda, G. Ghisellini, L. Nava, et al., The impact of selection biases on the $E_{\text {peak }}-L_{I S O}$ correlation of gamma-ray bursts, MNRAS 422 (2012) 2553.

[7] P. Jakobsson, A. Levan, J.P.U. Fynbo, et al., A mean redshift of 2.8 for Swift gamma-ray bursts, A\&A 447 (2006) 897.

[8] A. Melandri, B. Sbarufatti, P. D’Avanzo et al., The dark bursts population in a complete sample of bright Swift long gamma-ray bursts, MNRAS 421 (2012) 1265. 
[9] E. Nakar \& T. Piran, Outliers to the peak energy-isotropic energy relation in gamma-ray bursts, MNRAS 360 (2005) L73.

[10] L. Nava, G. Ghirlanda, G. Ghisellini, C. Firmani, Peak energy of the prompt emission of long gamma-ray bursts versus their fluence and peak flux, MNRAS 391 (2008) 639.

[11] L. Nava, G. Ghirlanda, G. Ghisellini, et al., Fermi/GBM and BATSE gamma-ray bursts: comparison of the spectral properties, MNRAS 415 (2011) 3153.

[12] L. Nava, R. Salvaterra, G. Ghirlanda, et al., A complete sample of bright Swift long gamma-ray bursts: testing the spectral-energy correlations, MNRAS 421 (2012) 1256 (N12).

[13] R. Salvaterra, S. Campana, S.D. Vergani, et al., A Complete Sample of Bright Swift Long Gamma-Ray Bursts. I. Sample Presentation, Luminosity Function and Evolution, ApJ 749 (2012) 68 (S12). 